\title{
Toxic Megacolon with Colonic Ischemia Masquerading as Diabetic Ketoacidosis: A Case Report
}

\author{
F. Mansouri
}

Department of Pediatrics, King Abdulaziz University, Jeddah, Saudi Arabia

DOI: $10.36348 /$ sjmps.2020.v06i01.010

| Received: 03.01.2020 | Accepted: 15.01.2020 | Published: 22.01.2020

*Corresponding author: Mansouri $\mathrm{F}$

\section{Abstract}

A previously healthy 12-year-old boy presented with abdominal pain and clinical and laboratory features highly suggestive of diabetic ketoacidosis. When his blood glucose plummeted and his urinary ketones disappeared within the first hour of insulin therapy, while his abdominal pain, acidosis and hemodynamic status failed to improve despite vigorous fluid resuscitation, the diagnosis of diabetic ketoacidosis was questioned. At laparotomy, gangrenous, hugely dilated large bowel was found, requiring a subtotal colectomy from the cecum to the sigmoid colon; leaving the patient with an ileostomy. The child survived a complicated postoperative course and is currently doing well.

Keywords: Ischemic bowel, toxic megacolon, diabetic ketoacidosis.

Copyright @ 2020: This is an open-access article distributed under the terms of the Creative Commons Attribution license which permits unrestricted use, distribution, and reproduction in any medium for non-commercial use (NonCommercial, or CC-BY-NC) provided the original author and source are credited.

\section{INTRODUCTION}

Twenty percent to $40 \%$ of children with newly diagnosed insulin-dependent (type-I) diabetes mellitus (IDDM) present with diabetic ketoacidosis (DKA)[1]. A common feature of DKA is acute abdominal pain, which usually improves with resolution of the ketonemia after fluid resuscitation and insulin therapy. This report describes a 12-year-old boy with abdominal pain and clinical and laboratory features highly suggestive of diabetic ketoacidosis in whom there was an abnormally rapid resolution of ketonuria and fall in blood glucose levels, with the abdominal pain, hemodynamic instability and acidosis not resolving as expected with fluid therapy and intravenous insulin. Owing to his deteriorating status and increasingly peritonitic abdomen, the patient underwent an exploratory laparotomy and was found to have extensive colonic necrosis with what appears to be a megacolon. This case is the first report of large bowel ischemia and toxic megacolon mimicking DKA in a child.

\section{Case Report}

A 12-year-old previously healthy boy presented to our emergency department (ED) with complaints of abdominal pain, nausea and vomiting for 4 days associated with subjective fever for 3 days. Prior to this, he had had a long-standing history of constipation and on and off abdominal pain. He had never been on any treatment and had been incompliant with dietary modifications. 4 days prior to presentation, he had progressively worsening episodes of abdominal pain, mainly in his right lower quadrant (RLQ). On the day of his presentation, he had severe, continuous abdominal pain, moderate amounts of non-bilious, nonbloody vomiting and subjective fever, without worsening of his constipation. He was brought to our ED by his family. He had a positive history of decreased appetite, polyuria and polydipsia, followed by a decrease in urine output and dysuria. He had no history of polyphagia or weight loss. He is fully vaccinated, has no past medical or surgical history and no recent travel. His younger sister is known to have IDDM.

Upon presentation, the patient looked ill. He appeared severely dehydrated, pale and had an altered level of consciousness (LOC). His vital signs in the ED were as follows: his heart rate ranged from 140-170 beats/minute, blood pressure was $85 / 30 \mathrm{mmHg}$, temperature was 37.7 degrees Celsius, respiratory rate was 40 breaths/minute, with an oxygen saturation of $99 \%$ in room air. His abdomen was rigid, tensely distended and tender all over with absent bowel sounds. His capillary refill time was 3 seconds, with weak peripheral pulses, acrocyanosis and cold extremities. His Glasgow Coma Scale (GCS) ranged from 12-13. His bedside blood glucose was $26 \mathrm{mmol} / \mathrm{L}$. 


\section{His initial laboratory investigations in the ED were as follows}

-A complete blood count (CBC) showed a white cell count of $19.48 \mathrm{~K} / \mathrm{uL}$, hemoglobin of 15.4 $\mathrm{g} / \mathrm{dL}$, a hematocrit of $49.8 \%$, a platelet count of 546 $\mathrm{K} / \mathrm{uL}$, a neutrophil count of $15.44 \mathrm{~K} / \mathrm{uL}(79.2 \%)$ and an immature granulocyte count of $0.26 \mathrm{~K} / \mathrm{uL}$.

-A venous blood gas (VBG) showed a $\mathrm{pH}$ of 6.80, a $\mathrm{PCO}_{2}$ of $3.42 \mathrm{KPa}, \mathrm{a} \mathrm{PO}_{2}$ of $4.16 \mathrm{KPa}$ and a bicarbonate level of $6.0 \mathrm{mmol} / \mathrm{L}$.

-His chemistries showed a sodium of 129 $\mathrm{mmol} / \mathrm{L}$, a potassium of $5.2 \mathrm{mmol} / \mathrm{L}$, a chloride of 94 $\mathrm{mmol} / \mathrm{L}$, a blood urea nitrogen $(\mathrm{BUN})$ of $15.7 \mathrm{mmol} / \mathrm{L}$, a creatinine level of $210 \mathrm{mmol} / \mathrm{L}$, a calcium level of 2.0 $\mathrm{mmol} / \mathrm{L}$, a phosphorus level of $4.3 \mathrm{mmol} / \mathrm{L}$ and a magnesium level of $2.01 \mathrm{mmol} / \mathrm{L}$.

-His liver function tests (LFT's) showed a total protein level of $77 \mathrm{~g} / \mathrm{L}$, a serum albumin level of 35 $\mathrm{g} / \mathrm{L}$, an alkaline phosphatase of $150 \mathrm{U} / \mathrm{L}$, aspartate aminotransferase (AST) of $105 \mathrm{U} / \mathrm{L}$, alanine aminotransferase (ALT) of $58 \mathrm{U} / \mathrm{L}$, gamma glutamyltransferase (GGT) of $13 \mathrm{U} / \mathrm{L}$, a total bilirubin of 26 umol/L, a serum lipase of $157 \mathrm{U} / \mathrm{L}$ and serum amylase of $79 \mathrm{U} / \mathrm{L}$.

-His coagulation profile revealed a prothrombin time (PT) of 34.3 seconds, a partial thromboplastin time (PTT) of 83.9 seconds, an International Normalized Ratio (INR) of 3.3, a serum fibrinogen level of $148 \mathrm{mg} / \mathrm{dL}$, and a D-dimer of 2.661 $\mathrm{ng} / \mathrm{ml}$.

-His C-reactive protein (CRP) was $13.1 \mathrm{mg} / \mathrm{L}$ and his serum lactate was $12 \mathrm{mmol} / \mathrm{L}$. A blood culture was also sent and showed no growth after 5 days of incubation.

-His urine was cola-colored and a very small amount was obtained with insertion of an indwelling catheter. A urinalysis (UA) revealed a highly concentrated urine and 3+ ketones, was negative for glucose, nitrite and leukocyte esterase, and had normal white and red blood cell counts. His urine culture was negative after 2 days of incubation. His stool was brown in color, and a stool analysis, microscopy and culture were all negative.

-Some additional laboratory tests that were performed included an insulin-like growth factor-1 (IGF-1) level of $41.1 \mathrm{ng} / \mathrm{ml}$, an insulin-like growth factor binding protein-3 (IGFBP-3) level of $2360 \mathrm{ng} / \mathrm{ml}$, fasting blood glucose of $22.5 \mathrm{mmol} / \mathrm{L}$, and a C-peptide level of $0.642 \mathrm{ng} / \mathrm{ml}$.

-His human immunodeficiency virus (HIV), hepatitis B surface antigen (HBsAg), hepatitis C virus
(HCV) antibody and methicillin-resistant Staphylococcus aureus (MRSA) tests were all negative.

Portable chest and abdominal X-rays were obtained in the ED. While the chest X-ray was largely unremarkable, the abdominal X-ray demonstrated abnormal bowel gas patterns and air fluid levels and what appeared to be a megacolon (Figures 1,2).

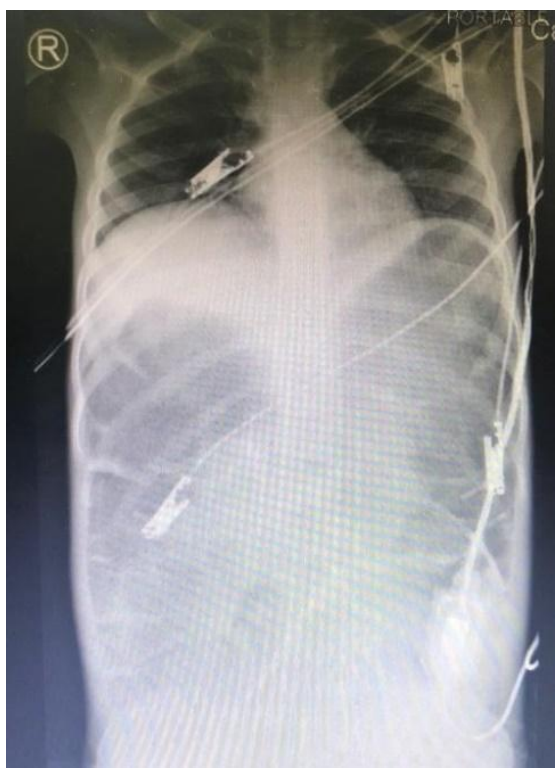

Fig-1: Portable chest and abdominal X-ray image obtained in the ED

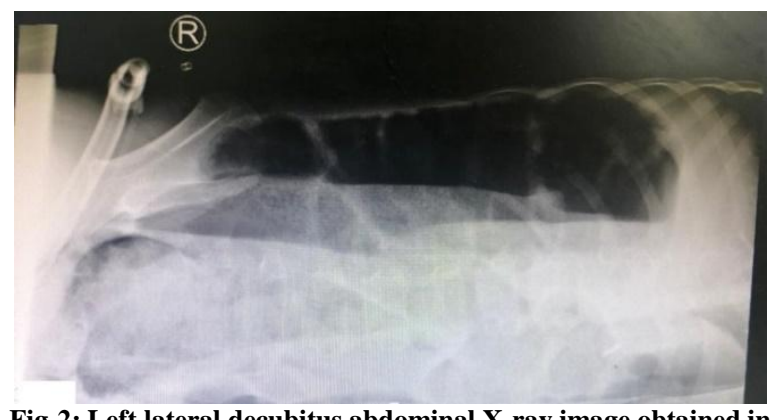

Fig-2: Left lateral decubitus abdominal X-ray image obtained in ED

An ultrasound of his abdomen and pelvis was performed urgently and was reported as: "Suboptimal examination as the entire abdomen demonstrates dirty shadowing, in keeping with a gassy abdomen".

Based on his clinical presentation and laboratory findings, in addition to a positive family history, the patient was managed as a case of DKA. After a discussion with endocrinology, our center's DKA protocol was initiated, and the patient received insulin infusion at $0.1 \mathrm{unit} / \mathrm{kg} / \mathrm{hour}$, with $0.9 \%$ saline at maintenance and $10 \%$ deficit to run over 48 hours. Prior to this, he had received two $10 \mathrm{ml} / \mathrm{kg}$ boluses of $0.9 \%$ saline, with the blood pressure only going up to $94 / 55$ $\mathrm{mmHg}$. The patient also received ceftriaxone $75 \mathrm{mg} / \mathrm{kg}$ and vancomycin $15 \mathrm{mg} / \mathrm{kg}$ for suspected sepsis. He 
received a full dose of vancomycin as his renal function was not available at the time of administration.

There was also suspicion of a surgical abdomen due to a rigid, tender and distended abdomen on physical exam and a grossly abnormal abdominal Xray. As such, pediatric surgery was consulted, who gave an impression of paralytic ileus with overflow incontinence, as the patient was still passing stool. The patient was kept nil per os (NPO) per their recommendation and a nasogastric tube (NGT) was inserted, from which a coffee-ground aspirate was obtained. He was started on metronidazole in addition to his antibiotics plus supportive care, and planned for a computed tomography (CT) scan of his abdomen when stable.

Nephrology was also consulted as the patient's renal function tests revealed evidence of acute kidney injury (AKI) and the decision was made to start continuous renal replacement therapy (CRRT) when the patient is more stable and his coagulation profile has normalized. His abnormal coagulative functions were thought to be likely due to disseminated intravascular coagulation (DIC) secondary to overwhelming sepsis. Owing to the patient's critical condition, it was decided that he be admitted to the pediatric intensive care unit (PICU).

Within one hour into his PICU admission, the patient's blood glucose level dropped rapidly, reaching $4.0 \mathrm{mmol} / \mathrm{L}$, at which point his insulin infusion was discontinued per discussion with endocrinology. This called the diagnosis of DKA into question, especially since ketones in his urine became negative after only 2 hours of initiating the DKA protocol. Around 30 minutes later, his blood glucose level plummeted even further, reaching $1.1 \mathrm{mmol} / \mathrm{L}$, at which point he started experiencing twitches of his right upper extremity, for which he was given a bolus of $10 \%$ dextrose at $4 \mathrm{ml} / \mathrm{kg}$. Shortly thereafter, the patient's blood pressure fell to $80 / 30 \mathrm{mmHg}$ and the patient went into shock once again, with tachycardia and altered LOC, disorientation and hallucinations. A left external jugular line was inserted and $500 \mathrm{ml}$ of $0.9 \%$ saline fast push was administered, as well as a lactated Ringer bolus as his blood glucose level was $3 \mathrm{mmol} / \mathrm{L}$. Despite fluid resuscitation, his blood pressure was not improving, so an epinephrine infusion was started at 0.1 $\mathrm{mcg} / \mathrm{kg} / \mathrm{minute}$ and titrated to effect. His antibiotic regimen was upgraded to meropenem and linezolid, in addition to metronidazole. The patient's urine output finally improved from 0.4 to $1.7 \mathrm{ml} / \mathrm{kg} /$ hour, so his fluids were decreased to maintenance plus $7 \%$ deficit over 48 hours.

With his condition relatively stabilized, the patient was sent for CT of his brain, which showed "abnormal hypodensity involving the subcortical white matter, thalami and external capsule, with brain swelling and effacement of the cortical sulci. No acute hemorrhage or definite infarction and no midline shift or mass effect". The patient received $3 \%$ saline $3 \mathrm{ml} / \mathrm{kg}$ in the PICU in response to these CT findings.

Upon his return to PICU, the patient deteriorated yet again, with worsening shock, tachypnea and LOC. He then developed a generalized tonic clonic seizure that lasted 30 seconds and was aborted with diazepam. He was ultimately intubated with a size 5 cuffed endotracheal tube and maintained on mechanical ventilation. The patient continued to be hypotensive with poor perfusion, so his epinephrine was increased to $0.4 \mathrm{mcg} / \mathrm{kg} / \mathrm{minute}$ and norepinephrine was added at 0.1 $\mathrm{mcg} / \mathrm{kg} / \mathrm{minute}$. Hydrocortisone $50 \mathrm{mg}$ was also given.

The patient had an echocardiogram performed, showing poor cardiac function, with an ejection fraction (EF) of $38 \%$ and a central venous pressure (CVP) of 12 $\mathrm{mm} / \mathrm{Hg}$. He was maintained on an infusion of furosemide $0.1 \mathrm{mg} / \mathrm{kg} / \mathrm{hour}$. To correct his coagulopathy, the patient received fresh frozen plasma (FFP) and cryoprecipitate. He also received multiple packed red blood cell (PRBC) and albumin transfusions.

Despite all efforts to maintain the patient's blood pressure with volume expanders and pressors, the patient remained hemodynamically unstable. It was on the next day that the patient was finally taken to the operating room (OR) by pediatric surgery. He underwent an exploratory laparotomy, which revealed a dilated colon that was dusky with blackish discoloration (Figures 3,4). There was no volvulus, perforation or fat creeping. Multiple lymph nodes were found. Subtotal colectomy was ultimately performed, leaving only the sigmoid colon and rectum, and the abdomen was kept open with a drain in place.

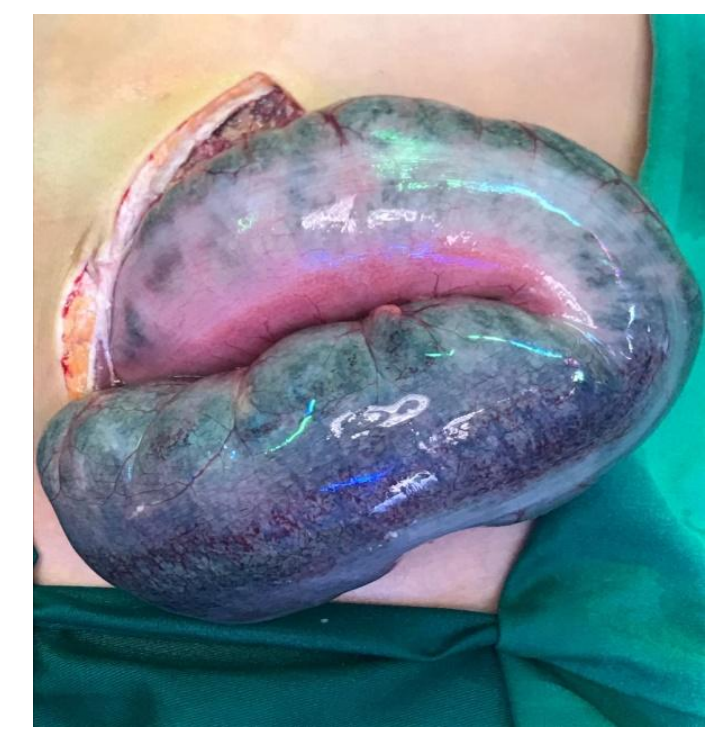

Fig-3: A dilated, dusky colon with blackish discoloration was found during exploratory laparotomy 


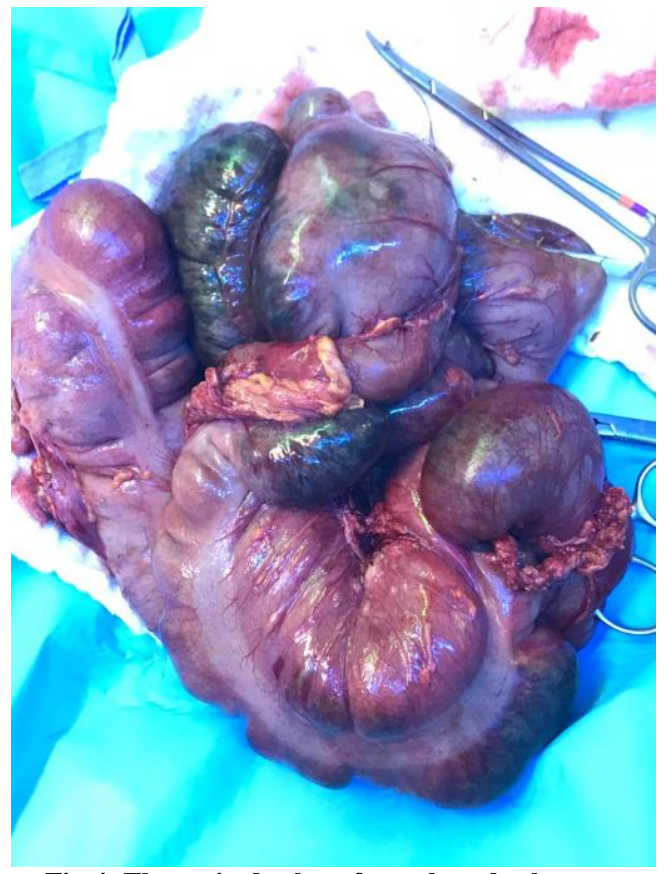

Fig-4: The excised colon after subtotal colectomy

The pathology report of the colonic specimen sent from the OR reads as follows: "Transmural intestinal necrosis consistent with ischemic bowel necrosis and focal perforation with reactive lymph node hyperplasia".

The patient's hemodynamic status and general condition began to improve after the operation. 5 days later, the patient was again taken to OR by pediatric surgery for a second exploratory laparotomy, where a right ileostomy was created and only abdominal skin was closed. Another 9 days later, a re-exploration was performed where a recto-sigmoid mucus fistula was created and rectal biopsy was sent, which demonstrated presence of ganglia. At this point, the abdomen was finally fully closed.

The patient had a prolonged, complicated course in the PICU before he was finally deemed suitable for transfer to the pediatric ward after 43 days in PICU. He was discharged with an impression of resolved septic shock due to possible toxic megacolon/bowel ischemia of unknown cause and significant weight loss. Issues during his PICU course included:

- Possible toxic megacolon vs. volvulus vs. adhesive bowel obstruction, the cause of which was never found. A CT angiography of the patient's abdomen revealed normal celiac, superior and inferior mesenteric circulations.

- Septic shock with multiorgan dysfunction syndrome (MODS), involving cardiovascular, renal, respiratory and coagulation (DIC) systems.

- Multiple surgeries, subtotal colectomy and right ileostomy.

- $\quad$ AKI requiring CRRT in PICU.
- During his PICU course, the patient developed seizures and was started on levetiracetam, maintained at $60 \mathrm{mg} / \mathrm{kg} / \mathrm{day}$, phenytoin 8 $\mathrm{mg} / \mathrm{kg} /$ day and topiramate $25 \mathrm{mg}$ twice a day.

- He also developed hypertension and was maintained on atenolol $57 \mathrm{mg}$ daily and amlodipine $5 \mathrm{mg}$ daily.

The patient's active issues on the ward were weight loss and malnutrition. His weight on admission was $34 \mathrm{~kg}$, which dropped to $19.7 \mathrm{~kg}$ upon discharge from PICU. The patient was ultimately discharged home in overall good condition after establishing a comprehensive nutritional regimen with our dietary services, and close follow up with general pediatrics, pediatric surgery, nephrology, dietetics among other services.

\section{DiSCUSSION}

DKA is a relatively common presenting feature of IDDM in children, occurring in $20 \%$ to $40 \%$ of cases $[1,2]$. DKA is a severe metabolic derangement characterized by hyperglycemia (serum glucose $>300$ $\mathrm{mg} / \mathrm{dL}$ ), ketonemia, and acidosis (arterial $\mathrm{pH}<7.30$, serum bicarbonate $<15 \mathrm{mEq} / \mathrm{l}$ ). The primary goals of the initial management of DKA are the restoration of intravascular volume and correction of electrolyte and acid-base imbalances. Most centers utilize controlled rates of rehydration with isotonic fluids to minimize the risk of severe cerebral edema. Whether this treatment protocol actually reduces the occurrence of cerebral edema is unclear [3-5]. Appropriate fluid, electrolyte, and insulin therapy should result in resolution of the hemodynamic, respiratory, gastrointestinal, and metabolic abnormalities. Persistence of acidosis or hemodynamic instability, particularly when the blood glucose level and ketonemia have resolved, should alert the clinician to the possibility of a concomitant illness or a complication.

Nausea, vomiting, and anorexia occur in $75 \%$ of patients with DKA [6]. Gastric dilatation and diminished motility related to ketosis, hypokalemia, acidosis, and hyperglycemia may account for some of the abdominal symptoms. Because the abdominal pain that often accompanies DKA usually improves and eventually resolves with fluid resuscitation and correction of the metabolic disturbances, persistent abdominal pain should alert physicians to the possibility of an accompanying abdominal process. In our case, it became abundantly clear that the patient, in fact, was not suffering from DKA, but rather a constellation of metabolic disturbances that were secondary to his intraabdominal pathology. It should be kept in mind that abdominal pathological processes may precipitate DKA in a child with undiagnosed or established IDDM. 
Dimeglio et al. [7] described a 3-year-old girl with massive ischemic intestinal necrosis at the onset of diabetes mellitus with ketoacidosis. In contrast to our patient, the child had new onset IDDM with severe acidosis and hypovolemia leading to poor tissue perfusion and subsequent ischemic necrosis. Our patient had clinical and laboratory findings that closely resembled those of DKA, but were only secondary to his intra-abdominal process. Although there have been multiple reports [8-11] of bowel ischemia and infarction in patients with established diabetes mellitus, there has been no previously reported case of intestinal necrosis in a child that presented in a manner nearly identical to that typical of DKA in whom the diagnosis of diabetes was challenged and ultimately refuted.

Megacolon is a descriptive term that defines dilatation of the colon that is not caused by mechanical obstruction $[12,13]$. The causes of megacolon can be classified into congenital and acquired. Patients with congenital megacolon usually experience onset of constipation in infancy and early childhood. The differential diagnosis of congenital megacolon includes visceral myopathies and enteric neuropathies such as Hirschsprung's disease and Waardenburg-Shah syndrome. A large proportion of patients with nonHirschsprung's congenital megacolon have the onset of symptoms in early childhood or adolescence and it appears to occur more commonly in males [14, 15]. Idiopathic megacolon describes an abnormality of the colon characterized by a permanent distension of the bowel lumen in the absence of an identifiable cause. In contrast to the well-defined histological abnormalities in Hirschsprung disease, the pathological basis underlying idiopathic megacolon is unknown. Possible mechanisms include abnormalities in the extrinsic nerves, the enteric nervous system, intestinal smooth muscle and neurotransmitters [16-18].

Idiopathic megacolon is a diagnosis of exclusion. Identification of electrolyte imbalances and thyroid dysfunction is important. Anorectal manometry may show lack of relaxation of the internal anal sphincter with balloon rectal distension. A normal rectoanal inhibitory reflex excludes the diagnosis of Hirschsprung disease, the diagnosis of which must be established by a rectal suction biopsy showing the absence of ganglion cells in the distal colon.

Many patients with idiopathic megacolon can be managed successfully using long-term laxatives, although some require surgery [19]. Surgical options include stoma formation, colectomy and anastomosis [20] and a variety of pull-through procedures [21]. Emergency surgery is indicated if there is evidence of toxic megacolon or volvulus.

Toxic megacolon (TM) is defined as an acute dilatation of the colon associated with clinical evidence of toxemia. It generally presents with abdominal distension, constipation, reduced bowel sounds and may be associated with fever, tachycardia, or hypotension. Although this is most frequently associated with ulcerative colitis, it may also occur in association with a variety of other conditions, including Crohn's disease, amebic colitis, pseudomembranous colitis, cholera, and bacillary dysentery. TM complicating Hirschsprung disease is quite rare[22].

The literature reports many cases of emergency surgery for toxic megacolon in association with ulcerative colitis, pseudomembranous colitis and Hirschsprung disease. It is rare in cases of idiopathic megacolon. Leventhal et al. [23] reported a case of toxic idiopathic megacolon with a fatal outcome in an intellectually disabled adolescent who also had pseudomembranous colitis. None of these reports, however, describe clinical features or laboratory findings that mimic those of DKA.

\section{Conclusion}

This report highlights a case of megacolon and intestinal necrosis of unclear cause that presented in a manner that closely mimicked DKA. Acidosis, hemodynamic instability, and abdominal pain that persist after appropriate fluid and insulin administration should alert physicians to the possibility of concurrent abdominal pathology. A high index of suspicion, careful abdominal examination, radiographic evaluation, and early surgical intervention, when indicated, are critical factors influencing survival.

\section{REFERENCES}

1. Dibua, U. M., Ugonabo, J. A. C., Oladepo, D., Iroha, I. R., \& Odimegwu, N. D. (2013). Genital Chlamydia and HIV co-infection: adverse pregnancy outcomes. American Journal of Research Communication, 1(12), 470-500.

2. Sperling, M. (1996). Diabetes mellitus, in Sperling M (ed): Pediatric Endocrinology. Toronto, Ontario, Saunders, 229-263

3. Sartor, G., Dahlquist, G. (1995). Short-term mortality in childhood onset insulin-dependent diabetes mellitus: A high frequency of unexpected deaths in bed. Diabetic Med, 12:607-611.

4. Klekamp, J., \& Churchwell, K. B. (1996). Diabetic ketoacidosis in children: initial clinical assessment and treatment. Pediatric Annals, 25(7), 387-393.

5. Mel, J. M., \& Werther, G. A. (1995). Incidence and outcome of diabetic cerebral oedema in childhood: are there predictors?. Journal of paediatrics and child health, 31(1), 17-20.

6. Goyal, R. K., \& Spiro, H. M. (1971). Gastrointestinal manifestations of diabetes mellitus. Medical Clinics of North America, 55(4), 1031-1044. 
F. Mansouri; Saudi J Med Pharm Sci, Jan., 2020; 6(1): 58-63

7. DiMeglio, L. A., Chaet, M. S., Quigley, C. A., \& Grosfeld, J. L. (2003). Massive ischemic intestinal necrosis at the onset of diabetes mellitus with ketoacidosis in a three-year-old girl. Journal of pediatric surgery, 38(10), 1537-1539.

8. Chan-Cua, S., Jones, K. L., Lynch, F. P., \& Freidenberg, G. R. (1992). Necrosis of the ileum in a diabetic adolescent. Journal of pediatric surgery, 27(9), 1236-1238.

9. Selby, C. D., Dennis, M. J., \& Whincup, P. H. (1987). Painless mesenteric infarction in patient with diabetes mellitus. Diabetes care, 10(2), 259259.

10. Sharieff, G. Q., Shad, J. A., \& Garmel, G. (1997). An unusual case of mesenteric ischemia in a patient with new-onset diabetes mellitus. The American journal of emergency medicine, 15(3), 282-284.

11. Nicol, K. K., \& Davis, G. J. (1997). An unusual complication of diabetes mellitus: the zebra that became a horse. Southern medical journal, 90(1), 83-85.

12. Camilleri, M. (2006). Acute and chronic pseudoobstruction. Sleisenger and Fordtran's Gastrointestinal and Liver Disease: Pathophysiology, Diagnosis, and Management (8th ed), Elsevier Saunders, Philadelphia, 2679-2702.

13. Camilleri, M. (2003). Dysmotility of the small intestine and colon. In: Yamada T, ed. Textbook of gastroenterology. 4th edn Vol 1 Philadelphia, USA: Lippincott Williams \& Wilkins, 1486-529

14. Barnes, P.R., Lennard-Jones, J.E., Hawley, P.R. (1986). Hirschsprung's disease and idiopathic megacolon in adults and adolescents. Gut, 27:53441

15. Gattuso JM, Kamm MA. Clinical features of idiopathic megarectum and idiopathic megacolon. Gut 1997;41:93-9

16. Gattuso, J.M., Kamm, M.A., Talbot, J.C. (1997). Pathology of idiopathic megarectum and megacolon. Gut, 41:252-7

17. Gattuso, J.M., Hoyle, C.H., Milner, P. (1996). Enteric innervation in idiopathic megarectum and megacolon. Int J Colorectal Dis. 11:264-71

18. Gattuso, J.M., Smith, V.V., Kamm, M.A. (1998). Altered contractile proteins and neural innervation in idiopathic megarectum and megacolon. Histopathology, 33:34-8

19. Kamm, M.A., Stabile, G. (1991). Management of idiopathic megarectum and megacolon. Br J Surg. 78:899-900

20. Stabile, G., Kamm, M.A., Hawley, P.R. (1991). Colectomy for idiopathic megarectum and megacolon. Gut, 32:1538-40

21. Marquez, T.T., Acton, R.D., Hess, D.J. (2009). Comprehensive review of procedures for total colonic aganglionosis. J Pediatr Surg, 44:257-65.

22. Khasanov, R., Schaible, T., Wessel, L.M., Haql, C.1. (2015). The Surgical Treatment of Toxic Megacolon in Hirschsprung Disease. Paediatr Emer Care.

23. Leventhal, A., Gimmon, Z. (1978). Toxic idiopathic megacolon: fatal outcome in a mentally retarded adolescent. Dis Colon Rectum. 21:383-6. 